\title{
Clinical Significance of CRP and IL-6 Levels During the Perioperative Period of Hip Arthroplasty for Aged Patients with Femoral Neck Fractures
}

Fulong Zhao

Beijing Luhe Hospital

Lijuan Guo

Chinese Emergency General Hospital

xuefei wang ( $\sim$ 1425496527@qq.com )

Beijing Luhe Hospital

Yakui Zhang

Beijing Luhe Hospital

Research article

Keywords: Serum, CRP, IL-6, Aged patient, Femoral neck fracture, Hip arthroplasty

Posted Date: May 22nd, 2020

DOI: https://doi.org/10.21203/rs.3.rs-29926/v1

License: (9) This work is licensed under a Creative Commons Attribution 4.0 International License.

Read Full License 


\section{Abstract}

Background Aged patients with femoral neck fractures underwent hip arthroplasty become prone to infection. And serum CRP and IL-6 might be sensitive indicators of inflammation. The aim of the study is to analyze and explore the clinical significance of changes in C-reactive protein (CRP) and interleukin-6 (IL-6) levels during the perioperative period in older patients that underwent hip arthroplasty for femoral neck fractures.

Methods This retrospective study analyzed data for 82 patients that underwent hip arthroplasty for femoral neck fractures from June 2018 to June 2019in our hospital. Of these, 39 patients underwent hemiarthroplasty (HA) and 43 underwent total hip arthroplasty (THA). CRP and IL- 6 were measured before the operation (baseline) and on the 1st, 2nd, 3rd, and 7th day after hip arthroplasty. Comparisons between groups were performed with the $t$ test and $\chi^{2}$ test.

Results Post-operative serum CRP and IL-6levels showed a trend of first increasing, then decreasing, but all levels were higher than baseline. The serum CRP level peaked on the third post-operative day, and IL- 6 peaked on the first post-operative day. We found no significant difference in CRP or IL-6 levels between patients that received HAs and patients that received THAs.

Conclusion We showed a trend in CRP and IL- 6 changes during the perioperative period. These findings suggested that CRP and IL- 6 might be involved in the early inflammatory response. Thus, both CRP and IL-6 levels might be useful for evaluating the postoperative inflammatory response in older patients with femoral neck fractures that undergo hip arthroplasty.

\section{Background}

Femoral neck fractures are common in older individuals. These fractures can lower the quality of life, and they are one of the main causes of death among older individuals [1]. Due to the relatively unique anatomical position of femoral neck fractures, they frequently block the blood supply to the femoral head, and they can lead to femoral head necrosis. Hip replacements, including hemiarthroplasty (HA) and total hip arthroplasty (THA), are common surgical procedures for repairing hip fractures. For femoral neck fractures, hip replacements provide better clinical outcomes in older individuals [2]. However, infection is a severe complication in hip replacements [3].

Under the influence of trauma, surgery, and other factors, older patients with femoral neck fractures experience severe traumatic reactions, which result in the release of large amounts of CRP and IL-6. These cytokines modify the immune function, and patients become prone to infection [4-7]. Understanding the changes in CRP and IL- 6 after surgery could be important in predicting infections in older patients with femoral neck fractures. This information might facilitate determinations of appropriate clinical measures for reducing mortality and disability. 
The present study aimed to investigate the dynamic changes in serum CRP and IL-6 levels after hip replacements in older patients with femoral neck fractures.

\section{Methods}

\section{Patients and samples}

We retrieved clinical data for 82 older patients with femoral neck fractures that underwent hip arthroplasties in our hospital, from June 2018 to June 2019.This cohort included 33 males and 49 females, with an average age of $74.26 \pm 9.15$ years (range: 65 to 85 ). Femoral neck fractures occurred on the left side in 37 patients, and on the right side in 45 patients. All femoral neck fractures were recent, confirmed with CT. We excluded cases of old or pathological fractures. Among 39 patients that underwent HAs, prostheses were fixed with bone cement in 21 patients, and cement less (biological) prostheses were used in 18 patients. Among 43 patients that underwent THAs, prostheses were fixed with bone cement in 20 patients and biological prostheses were used in 23 patients. These two treatment groups (i.e., bone cement and biological fixations) were not significantly different in patient characteristics, including age, gender, BMI, and postoperative pain.

In the morning, on an empty stomach, 2 milliliters of venous blood were collected from patients one day before the operation and on the first, third, and seventh days after the operation. Serum was isolated by centrifuging at $3000 \mathrm{rpm} / \mathrm{min}, 4^{\circ} \mathrm{C}$, for $20 \mathrm{~min}$. Samples were stored at $-70^{\circ} \mathrm{Cbefore}$ testing. Serum CRP and IL-6 levels were measured with an enzyme-linked immunosorbent assay (ELISA; R\&D Systems, Camarillo, CA, USA).

\section{Statistical analysis}

All statistical analyses were performed with SPSS 19.0(SPSS Inc., Chicago, IL, USA). Data are reported as the mean \pm standard deviation (SD). Groups were compared with the $t$ test and $\chi^{2}$ test, as appropriate. Pvalues $\leq 0.05$ were considered significant.

\section{Results}

The THA and HA groups showed no significant differences in CRP or IL-6 levels, regardless of whether fixation was achieved with bone-cement or biologically.

Compared to preoperative levels, CRP levels were significantly elevated on the first through seventh days after surgery. CRP peaked on the third day after surgery and decreased significantly on the seventh day after surgery (Table 1$)$. 
Table 1

Serum CRP before and after hip replacement surgery in older patients with femoral neck fractures; prostheses were fixed with bone cement or biological fixation

\begin{tabular}{|c|c|c|c|c|}
\hline \multirow{2}{*}{$\begin{array}{l}\text { Time of CRP } \\
\text { measurement }\end{array}$} & \multicolumn{2}{|l|}{$\mathrm{HA}$} & \multicolumn{2}{|l|}{ THA } \\
\hline & Cement & Biological & Cement & Biological \\
\hline One day before surgery & $9.04 \pm 1.31$ & $8.92 \pm 0.95$ & $8.79 \pm 0.53$ & $8.68 \pm 1.43$ \\
\hline \multicolumn{5}{|l|}{ Post surgery } \\
\hline First day & $44.92 \pm 0.53^{*}$ & $46.48 \pm 1.82^{\star}$ & $45.01 \pm 1.97 *$ & $44.97 \pm 1.26^{\star}$ \\
\hline Second day & $85.01 \pm 2.57^{\star \Delta}$ & $86.44 \pm 1.85^{\star \Delta}$ & $83.64 \pm 0.81 * \Delta$ & $85.14 \pm 3.11 * \Delta$ \\
\hline Third day & $\begin{array}{l}113.82 \pm \\
1.43^{* \circ}\end{array}$ & $115.49 \pm 3.58^{*^{\circ}}$ & $116.09 \pm 2.35^{\star^{\circ}}$ & $112.48 \pm 1.63^{*^{\circ}}$ \\
\hline Seventh day & $44.29 \pm 3.29 *$ & $43.09 \pm 1.17^{\star}$ & $42.66 \pm 2.15^{\star}$ & $40.87 \pm 2.72^{\star}$ \\
\hline
\end{tabular}

Compared to the preoperative level, IL-6 increased significantly on the first through seventh days after surgery. IL-6 reached its peak on the first day after surgery, and gradually decreased on the second, third, and seventh days after surgery (Table 2 ).

Table 2

Serum IL-6 levels before and after hip replacement surgery in older patients with femoral neck fractures; prostheses were fixed with bone cement or biological fixation.

\begin{tabular}{|c|c|c|c|c|}
\hline \multirow[t]{2}{*}{ Time of IL- 6 measurement } & \multicolumn{2}{|l|}{ HA } & \multicolumn{2}{|l|}{ THA } \\
\hline & Cement & Biological & Cement & Biological \\
\hline One day before surgery & $8.94 \pm 1.17$ & $8.90 \pm 0.76$ & $9.15 \pm 0.34$ & $9.12 \pm 1.08$ \\
\hline \multicolumn{5}{|l|}{ Post surgery } \\
\hline First day & $153.12 \pm 1.33^{*}$ & $149.78 \pm 2.55^{\star}$ & $148.16 \pm 1.71 *$ & $150.09 \pm 2.39 *$ \\
\hline Second day & $94.91 \pm 3.01 * \Delta$ & $97.20 \pm 2.54^{\star \Delta}$ & $93.85 \pm 2.42 \star \Delta$ & $95.02 \pm 1.88 * \Delta$ \\
\hline Third day & $34.27 \pm 1.6 *^{\star \circ}$ & $33.95 \pm 2.35^{\star^{\circ}}$ & $35.14 \pm 0.99 \star^{\circ}$ & $33.92 \pm 2.82^{*^{\circ}}$ \\
\hline Seventh day & $15.18 \pm 2.56^{\star \dagger}$ & $18.11 \pm 3.63^{*^{\dagger}}$ & $14.40 \pm 2.34^{\star \dagger}$ & $16.17 \pm 1.35^{\star^{\dagger}}$ \\
\hline
\end{tabular}

Values are the mean \pm standard deviation. HA: hemiarthroplasty; THA: total hip arthroplasty; ${ }^{*} \mathrm{P}<0.05$ compared tolL- 6 on the day before surgery; ${ }^{\mathrm{P}} \mathrm{P}<0.05$ compared tolL- 6 on the first day after surgery; $\mathrm{P}<$ 0.05 compared tolL- 6 on the second day after surgery; ${ }^{\dagger} \mathrm{P}<0.05$ compared tolL- 6 on the third day after surgery. 


\section{Discussion}

Hip replacement is an effective treatment for femoral neck fractures in older individuals. It can improve limb function and quality of life. Patients often experience surgical stress reactions, particularly during the perioperative period $[8,9]$. Postoperative serum CRP and IL- 6 might be sensitive indicators of perioperative stress [10] and postoperative inflammation [11-13].Therefore, it is important to monitor serum CRP and IL-6 levels in older patients that undergo hip replacements for femoral neck fractures.

In the present study, we measured CRP and IL-6 in older patients with femoral neck fractures before and after surgery. To the best of our knowledge, this study was the first to demonstrate changes in CRP and IL-6 levels during the perioperative period of hip arthroplasty for femoral neck fractures in older patients. In this study, we investigated differences in CRP and IL-6 levels measured before and after surgery.

CRP can regulate inflammatory and immune responses in many biological settings. A type of acutephase protein, CRP is very low in normal states, but it can be induced by trauma and inflammation [14].This behavior was verified in this study. Compared to preoperative levels, postoperative CRP levels increased. Interestingly, after surgery, CRP levels increased and peaked on the third day in both the HA and THA groups. Our observations of perioperative CRP changes were consistent with findings in previous studies $[15,16]$.

IL-6 is mainly secreted by hepatocytes, macrophages, plasma cells, and so on. IL-6 is the key mediator for the inflammatory response and for host defense mechanisms [17]. IL- 6 can promote angiogenesis, and it is conducive to fracture healing [18]. Our results showed that, compared to preoperative levels, postoperative IL- 6 increased, and it peaked on the first day after surgery. These results suggested that IL- 6 was involved in early postoperative wound repair. The changes we observed in IL-6 during the perioperative period were consistent with some previous studies [19, 20].

Despite significantly increases in CRP andIL-6levels after surgery, these changes were not significantly different between the THA and HA groups. This finding indicated that THA may not increase the risk of trauma and surgery compared to HA. Our results also showed no correlation between CRP and IL-6 in any group, even though CRP is regulated by IL-6[21]. Additionally, in both the HA and THA groups, the IL-6 or CRP levels were similar between the two fixation groups (biological and bone-cement). This result indicated that bone cement had little effect on trauma and tissue repair.

This study had some limitations. First, our results were based on a small number of patients (82 patients). Second, we did not include healthy people or patients with infections after a hip replacement. Third, we could not accurately predict complications. In future, a large clinical case dataset should be studied to achieve reasonable, quantitative ranges of CRP and IL-6.

\section{Conclusion}


In conclusion, we found that both CRP and IL-6levelsincreased after surgery compared to before surgery. In addition, this study showed a trend of CRP and IL- 6 changes within 7 days after surgery. These results implicated that both CRP and IL-6levels might be useful for evaluating postoperative inflammatory responses in older patients with femoral neck fractures that undergo hip arthroplasty.

\section{Declarations}

\section{Ethics approval and consent to participate}

Written informed consent was obtained from patients under a protocol approved by the Ethics Committee of Beijing Luhe Hospital affiliated to Capital Medical University.

\section{Consent for publication}

Not applicable.

\section{Availability of data and materials}

All data analyzed during this study are included in this published article.

\section{Competing interests}

The authors declare that they have no competing interests.

\section{Funding}

No fund was received.

\section{Authors' contributions}

FL $Z$ and XF W designed and initiated the study. FL $Z$ and $Y K Z$ collected data. LJ G and XF W performed the statistical analysis. FL Z and LJ G wrote the article.

\section{Acknowledgements}

Not applicable.

\section{References}

1. Alexiou KI, Roushias A, Varitimidis SE, Malizos KN. Quality of life and psychological consequences in elderlypatients after a hip fracture: a review. Clin Interv Aging. 2018;13:143-50.

2. Florschutz AV, Langford JR, Haidukewych GJ, Koval KJ. Femoral neck fractures: current management. J Orthop Trauma. 2015;29(3):121-9. 
3. Parvizi J, Adeli B, Zmistowski B, Restrepo C, Greenwald AS. Management of periprosthetic joint infection: the current knowledge: AAOS exhibit selection.J Bone. Joint Surg Am. 2012;94(14):e104.

4. Nightingale S, Holmes J, Mason J. House A.Psychiatric illness and mortality after hip fracture.Lancet. 2001; 357(9264):1264-1265.

5. Kragsbjerg P, Holmberg H, Vikerfors T. Serum concentrations of interleukin-6, tumour necrosis factoralpha, and C-reactive protein in patients undergoing major operations. Eur J Surg. 1995;161(1):1722.

6. Kruidenier J, Dingemans SA, Van Dieren S, De Jong VM, Goslings JC, Schepers T. C-reactive protein kinetics and its predictive value in orthopedic (trauma) surgery: A systematic review. Acta Orthop Belg. 2018;84(4):397-406.

7. Slaats J, Ten Oever J, van de Veerdonk FL. Netea MG.IL-1 $\beta /$ IL-6/CRP and IL-18/ferritin: Distinct Inflammatory Programs in Infections.PLoS Pathog.2016; 12(12):e1005973.

8. Manou-Stathopoulou V, Korbonits M, Ackland GL. Redefining the perioperative stress response: a narrative review. Br J Anaesth. 2019;123(5):570-83.

9. Hirsiger S, Simmen HP, Werner CM, Wanner GA, Rittirsch D. Danger signals activating the immune response after trauma. Mediators Inflamm. 2012;2012:315941.

10. Kehlet $\mathrm{H}$, Binder $\mathrm{C}$. Adrenocortical function and clinical course during and after surgery in unsupplemented glucocorticoid-treated patients. Br J Anaesth. 1973;45(10):1043-8.

11. Demura S, Takahashi K, Kawahara N, Watanabe Y, Tomita K. Serum interleukin-6 response after spinal surgery: Estimation of surgical magnitude. J Orthop Sci. 2006;11:241-7.

12. Hall GM, Peerbhoy D, Shenkin A, Parker CJ, Salmon P. Relationship of the functional recovery after hip arthroplasty to the neuroendocrineand inflammatory responses. $\mathrm{Br} J$ Anaesth. 2001;87:537-42.

13. Watt DG, Horgan PG. McMillan DC.Routine clinical markers of the magnitude of the systemic inflammatory response after elective operation: a systematic review. Surgery. 2015;157(2):362-80.

14. Elgeidi A, Elganainy AE, Abou Elkhier N, Rakha S. Interleukin-6 and other inflammatory markers in diagnosis of periprosthetic joint infection. Int Orthop. 2014;38(12):2591-5.

15. Glehr M, Friesenbichler J, Hofmann G, Bernhardt GA, Zacherl M, Avian A, Windhager R, Leithner A. Novel biomarkers to detect infection in revision hip and knee arthroplasties. Clin Orthop Relat Res. 2013;471(8):2621-8.

16. Ikeuchi M, Kamimoto Y, Izumi M, Fukunaga K, Aso K, Sugimura N, Yokoyama M, Tani T. Effects of dexamethasone on local infiltration analgesia in total knee arthroplasty: a randomized controlled trial. Knee Surg Sports Traumatol Arthrosc. 2014;22:1638-43.

17. Abou El-Khier NT, El GAel-R, Elgeidy, Rakha A. SA. Assessment of interleukin-6 and other inflammatory markers in the diagnosis of Egyptian patients with periprosthetic joint infection. Egypt J Immunol. 2013;20(2):93-9.

18. Glass GE, Chan JK, Freidin A,et al. TNF-alpha promotes fracture repair by augmenting the recruitment and differentiation of muscle-derived stromall cells. Proc Natl Acad Sci USA. 2011;108(7):1585-90. 
19. Chen XX, Wang T, Li J, Kang H. Relationship between Inflammatory Response and Estimated Complication Rate after THA. Chin Med J (Engl). 2016;129(21):2546-51.

20. Zhong J, Si HB, Zeng Y, Yang J, Zhou ZK, Kang PD, Pei FX, Shen B. Comparison of cortisol and inflammatory response between aged and middle-aged patients undergoing THA: a prospective observational study. BMC Musculoskelet Disord. 2017;18(1):541.

21. Li Q, Xu W, Xue X, Wang Q, Han Lu, Li W, Lv S, Liu D, Richards J, Shen Z, Ma L, Qing Song. Presence of multimeric isoforms of human C-reactive protein in tissues and blood. Mol Med Rep. 2016;14(6):5461-6. 